\title{
Transtornos parafílicos em pacientes com transtorno obsessivo-compulsivo: série de casos
}

Paraphilic disorders among patients with obsessivecompulsive disorder: case series

Manuela C. Borges', Lídia Ordacgi', Rafael F. Garcia', Bruno P. Nazar', Leonardo F. Fontenelle’

\section{RESUMO}

Com o intuito de elucidar a relação entre transtornos do controle de impulsos (TCI) e transtorno obsessivo-compulsivo (TOC), faz-se mister estudar subgrupos mais clinicamente homogêneos de transtornos impulsivos. Por meio do relato de quatro casos de pacientes com TOC e diferentes tipos de transtornos parafílicos (fetichismo transvético, sadismo, ginandromorfofilia e exibicionismo), são discutidos os conceitos de compulsividade, impulsividade e a relação temporal entre ambos. O estudo dos casos aqui descritos mostra que (1) pacientes com TOC e transtornos parafílicos tendem a desenvolver o TOC primeiro, (2) diante de desejos, fantasias ou atos sexuais parafílicos, pacientes com TOC podem lançar mão de comportamentos tipicamente compulsivos, (3) pacientes com TOC e obsessões sexuais egodistônicas podem desenvolver desejos, fantasias ou atos sexuais parafílicos de conteúdo semelhante ao das obsessões, (4) em um mesmo paciente, TOC e parafilias podem apresentar cursos independentes, e (5) pacientes com TOC e parafilias podem não apresentar obsessões sexuais. O sofrimento de pacientes com TOC e parafilias justifica a investigação continuada de tais condições no intuito de elucidar os mecanismos que subjazem esta associação e de criar estratégias que aumentem a adesão ao tratamento.

\section{ABSTRACT}

In order to elucidate the relationship between impulse control disorders and obsessive-compulsive disorder (OCD), it is essential to study more clinically homogenous subgroups of patients with impulsive disorders. Using four cases of patients with OCD and comorbid paraphilias (transvestic fetishism, sadism, gynandromorphophilia, and exhibitionism) as reference-points, we discuss the concepts of compulsivity, impulsivity, and the temporal relationship between them. The case studies here described suggest that (1) patients with OCD and comorbid paraphilias tend to develop OCD first, (2) once developing paraphilic fantasies, desires, or behaviors, patients with OCD can exhibit typical compulsive behaviors in an attempt to keep these phenomena under control, (3) patients with OCD and ego-dystonic sexual obsessions can develop paraphilic fantasies, desires, or behaviors with similar content to the first phenomenon, (4) OCD and paraphilias can follow independent courses in the same patient, and (5) patients with OCD and paraphilias may not present obsessions with sexual content. The distress presented by patients with OCD and paraphilias give good reason for the continuous investigation of this association, aiming at clarifying the neurobiological mechanisms underlying this association.

1 Programa de Ansiedade e Depressão do Instituto de Psiquiatria da Universidade Federal do Rio de Janeiro (Ipub/UFRJ). 


\section{INTRODUÇÃO}

A existência de um espectro obsessivo-impulsivo, com o transtorno obsessivo-compulsivo (TOC) em um de seus extremos e os transtornos de controle do impulso (TCI) no pólo oposto, tem sido objeto de intensa discussão na literatura (Hollander e Wong 1995). Esse espectro englobaria transtornos que apresentam em comum uma incapacidade para postergar ou inibir comportamentos repetitivos, e uma resposta supostamente positiva ao tratamento com inibidores da recaptação de serotonina (IRS). Segundo o modelo hipotético proposto por Hollander e Wong (1995), o TOC seria caracterizado por hiperfrontalidade, aumento da função serotoninérgica e comportamentos repetitivos conduzidos, com o objetivo de evitar acontecimentos desagradáveis. Já os TCl estariam associados à hipofrontalidade, diminuição da função serotoninérgica e comportamentos repetitivos que resultam eventos desagradáveis.

Os transtornos parafílicos (ou simplesmente parafilias) são caracterizados por desejos, fantasias ou atos sexuais que envolvem a humilhação ou o sofrimento do parceiro, crianças ou pessoas desavisadas, ou objetos não-humanos. Segundo a DSM-IV-TR, as parafilias são exemplos de transtornos impulsivos classificados em outro local, isto é, junto aos transtornos sexuais. Uma análise superficial do modelo de Hollander e Wong (1995) e seu antagonismo entre compulsividade e impulsividade sugere que a associação entre TOC e TCl, em geral, e parafilias, em particular, é rara. No entanto, diversos estudos demonstram prevalências de transtornos de controle dos impulsos em pacientes com TOC que variam entre 16,4\% (Grant et al., 2006), 29,0\% (Matsunaga et al., 2005) e 35,5\% (Fontenelle et al. 2005).

A heterogeneidade destes achados reflete os diferentes conceitos de $\mathrm{TCl}$ adotados nestes estudos. Por exemplo, enquanto Grant et al. (2006) avaliaram predominantemente $\mathrm{TCl}$ não classificados em outro lugar (de acordo com a DSM-IV-TR), Fontenelle et al. (2005) e Matsunaga et al. (2005) adotaram conceitos mais amplos, incluindo neste grupo pacientes com abuso de álcool, bulimia nervosa e parafilias (Fontenelle et al., 2005), ou mesmo transtornos de personalidade borderline e anti-social e "impulsividade sexual" (Matsunaga et al., 2005).

Não há dúvidas de que o conceito de TCl é demasiadamente amplo. Portanto, com o intuito de elucidar a relação entre $\mathrm{TCl}$ e TOC, faz-se mister estudar subgrupos mais clinicamente homogêneos de transtornos impulsivos. Recentemente, por exemplo, Stewart et al (2005) constataram que pacientes com TOC e tricotilomania (um transtorno de controle dos impulsos não classificado em outro local) caracterizaram-se por uma predominância do sexo feminino, por apresentarem pelo menos um tique comórbido, TOC de início precoce, número inferior de obsessões de contami- nação e compulsões de verificação, e por encontrarem-se em uso de psicoestimulantes ou venlafaxina. No presente estudo, descrevemos quatro pacientes com uma associação entre TOC e transtornos parafílicos e discutimos a psicopatologia destas condições à luz do chamado espectro compulsivo-impulsivo.

\section{CASO 1}

Sr. A, um paciente do sexo masculino, 24 anos, negro, solteiro e bibliotecário, apresentou os primeiros sintomas obsessivo-compulsivos aos 16 anos, quando começou a sentir necessidade de pisar quatro vezes no pé da porta, antes de entrar. Pouco depois, desenvolveu obsessões de cunho sexual envolvendo seu pai (por exemplo, "ele quer que eu fique louco" e "ele quer transar comigo") e outros pensamentos repetitivos e desagradáveis que incluíam dúvidas como "será que eu tirei a virgindade das minhas irmãs?" e "será que eu tive orgasmo com o empregado homossexual lá de casa?".

Em resposta a esses pensamentos, o Sr. A executava comportamentos como piscar oito vezes, repetir o próprio pensamento e cruzar a perna por quatro vezes. Outras compulsões presentes incluíam a necessidade de subir e descer de sua moto duas vezes, cantar uma música para "ficar protegido" e pisar de uma maneira especial por cinco vezes, cada vez que pisava em falso, por achar que isso era "coisa de homossexual".

Dois anos mais tarde, o Sr. A passou a usar roupas íntimas das irmãs durante a masturbação. Sempre tentava atingir um total de quatro episódios masturbatórios, que eram seguidos por muita culpa. Depois da masturbação, o paciente não só feria seus braços e pernas, como forma de expiação, também realizava outros comportamentos compulsivos descritos anteriormente, como a repetição de rituais um determinado número de vezes. Seus diagnósticos foram TOC e fetichismo transvéstico.

Entre as drogas utilizadas pelo Sr. A, por dose e tempo adequados, encontravam-se fluoxetina e paroxetina potencializadas por risperidona, tioridazina e carbamazepina. Após ser admitido em nosso serviço sem medicações, o paciente fez uso de fluoxetina $80 \mathrm{mg}$ e alprazolam 2 mg, sem apresentar melhora. Cerca de quatro meses depois, o paciente abandonou o tratamento.

\section{CASO 2}

Sr. B, um paciente do sexo masculino, 44 anos, branco, casado e dentista, apresentou os primeiros sintomas aos 6 anos, quando começou a fazer gestos obscenos, a piscar e a bater na parte superior das portas. Desde aquela época, é tomado 
por uma imagem em que é violentado sexualmente e pela idéia de que seus parentes seriam aleijados, cegos e doentes.

$\mathrm{Na}$ época de admissão em nosso serviço, apresentava tiques faciais, como piscar excessivamente e mostrar a língua, e fônicos, como fungar, tossir e pronunciar palavras obscenas. Tiques complexos, como girar os braços, também estavam presentes. Além disso, mantinha obsessões somáticas e de agressão.

Desde os 18 anos, o Sr. B apresentava fantasias sexuais nas quais era um executivo que espanca, humilha e abusa de sua secretária. As fantasias eram acompanhadas por masturbação. O paciente imaginava tais situações e se masturbava todos os dias antes de dormir, mesmo com a esposa ao lado, o que interferia significativamente em seu relacionamento marital. Os diagnósticos do Sr. B foram síndrome de La Tourette, TOC e sadismo sexual.

O paciente procurou tratamento pela primeira vez aos 26 anos. Ao longo de sua vida, Sr. B fez uso de vários medicamentos, incluindo risperidona, clonazepam, flufenazina, pimozida e bromazepam. Em nosso serviço, o paciente foi tratado com quetiapina em doses de até $100 \mathrm{mg} / \mathrm{dia}$. Sr. B relatou melhora parcial dos pensamentos obsessivos após quatro semanas. No entanto, o desenvolvimento de sonolência impediu acréscimos adicionais da dose. Durante todo o seguimento, suas fantasias sexuais se mantiveram inalteradas e os tiques evoluíram com flutuações importantes. O paciente abandonou o tratamento após cerca de seis meses.

\section{CASO 3}

Sr. C, um paciente do sexo masculino de 40 anos, pardo, solteiro e digitador, apresentou os primeiros sintomas aos 8 anos, quando começou a "falar para encher o tempo". Esse sintoma persiste até hoje, e enquanto o Sr. C não completa o raciocínio, repete as sílabas "sé, sé, sé, sé...".

Ao ser admitido em nosso serviço, o paciente ainda apresentava obsessões de contaminação, como preocupação com sujeira e micróbios e vasto número de compulsões, como contar as próprias fezes, interromper a evacuação para dar descarga, lavar cada parte do corpo sete vezes e alinhar quadros em sua parede. O Sr. C também colecionava objetos, como copos, miniaturas, animais empalhados, figurinhas, bonecos, discos, CDs e placas de trânsito.

O paciente apresentava, desde o início da idade adulta, fantasias envolvendo homens com aspectos feminilizados (travestis), com quem ocasionalmente mantinha contato sexual. O Sr. C permanecia horas perambulando pelas ruas onde os travestis trabalhavam durante a noite. Sentia-se profundamente atraído pela curvas dos travestis e essa fantasia não o preocupava. Dizia ser "um garanhão" e ter muitas parceiras. Seus diagnósticos foram transtorno de tique fônico crônico, TOC e ginandromorfofilia.
Em nosso serviço, o paciente foi medicado com fluoxetina, em doses de até $60 \mathrm{mg} / \mathrm{dia}$. Embora tenha apresentado melhora parcial dos sintomas obsessivo-compulsivos, o paciente desenvolveu náusea, o que motivou a interrupção do tratamento várias vezes, com piora dos sintomas. Foi então medicado com clomipramina, até $50 \mathrm{mg} / \mathrm{dia}$, mas o paciente passou a se queixar de sonolência e cansaço, o que o levou a abandonar o tratamento após menos de três meses. Durante todo o período de seguimento, o quadro de ginandromorfofilia permaneceu inalterado.

\section{CASO 4}

Sr. D, um paciente do sexo masculino de 22 anos, negro, solteiro e professor, apresentou as primeiras compulsões de simetria e organização aos 13 anos. Relatava organizar seus livros em casa cuidadosamente e empilhá-los sempre da mesma maneira antes de iniciar uma aula. Sr. D afirmava ainda ter em casa todos os testes e provas que já fez durante a vida organizados em pastas. Percebia um odor desagradável originário de suas axilas ao fim da jornada de trabalho. Esse odor não era perceptível para as outras pessoas. O paciente evitava se envolver em atividades sociais imediatamente depois do trabalho, pois acreditava que esse cheiro poderia incomodar e constranger seus colegas. Além disso, ao dormir em companhia de sua namorada, acordava no meio da noite para tomar banho e passar desodorante.

Aos 18 anos, passou a exibir seus genitais a mulheres desavisadas. O paciente gastava horas perambulando pelas ruas, todos os dias, em busca de uma situação que possibilitasse o comportamento exibicionista. Dizia perder noites inteiras procurando uma oportunidade para exibir sua genitália. Preferia exibir seus genitais a mulheres que se vestissem "como piranhas" e ficava excitado, tanto com reações de repulsa quanto de curiosidade por parte das mulheres. Afirmava atingir o orgasmo invariavelmente ao notar que sua vitima olhava seu pênis. O paciente masturbava-se repetidamente pensando nas mulheres a quem já havia se exibido. Os diagnósticos do Sr. C foram TOC e exibicionismo.

Ao ser admitido em nosso serviço, foi medicado com venlafaxina, $75 \mathrm{mg} / \mathrm{dia}$, com melhora discreta dos sintomas obsessivo-compulsivos ao final de um mês. O quadro de exibicionismo, no entanto, permaneceu inalterado. Logo em seguida, o paciente abandonou o tratamento.

\section{DISCUSSÃO}

Embora diversos estudos apontem para uma associação entre o TOC e os TCl, a heterogeneidade dos TCl dificulta enormemente a elucidação das relações entre tais condições, tanto do ponto de vista dos sintomas quanto do curso, da resposta ao 
tratamento e dos substratos neurais subjacentes. O estudo de populações mais homogêneas do ponto de vista clínico pode ajudar de maneira significativa na compreensão da complexa relação entre pacientes com TOC e TCI. Daí a importância de investigar a relação entre o TOC e um TCl em específico, por exemplo, as parafilias. Nossa série de casos aqui relatada pode representar um importante passo neste sentido.

Os casos aqui descritos sugerem que, quando ocorre uma associação entre o TOC e transtornos parafílicos, o TOC tende a surgir primeiro. De fato, este curso de aparecimento dos sintomas parece refletir o período habitual de desenvolvimento dos transtornos psiquiátricos em análise. Em outras palavras, pacientes com TOC costumam apresentar início precoce ou até mesmo pré-puberal dos sintomas (Rosário-Campos et al., 2001; Fontenelle et al., 2003), enquanto pacientes com parafilias só apresentam os sintomas deste transtorno quando se encontram em um estágio mais avançado do desenvolvimento psicossexual (Grant, 2005). A relação temporal entre os transtornos psiquiátricos aqui descritos também contradiz a hipótese de que o TOC possa ser uma condição secundária às parafilias, ou seja, uma tentativa de suprimir desejos, fantasias ou atos sexuais inaceitáveis (Stein, 1994).

No entanto, o paciente já portador de TOC, uma vez desenvolvendo uma parafilia, pode lançar mão de comportamentos compulsivos na tentativa de anular ou de manter seus desejos, fantasias ou atos sexuais sob controle. Por exemplo, no caso 1, o Sr. A utilizava roupas íntimas da irmãs durante a masturbação e, logo após o orgasmo, feria seus braços e pernas como forma de expiação, além de repetir atividades rotineiras determinado número de vezes. De fato, existem alguns relatos na literatura que sugerem que fantasias parafilicas podem apresentar características egodistônicas, como aquelas presentes em um pensamento tipicamente obsessivo (Abdo et al., 2001).

Uma outra possibilidade seria a de que um paciente com TOC com imagens sexuais egodistônicas pudesse se sentir sexualmente atraído pelo conteúdo de tais vivências mediante sua repetição e habituação. Nesta linha, o Sr. B (caso 2) apresentava, na infância, imagens nas quais ele mesmo era violentado e seus parentes aleijados, cegos e doentes. Posteriormente, aos 18 anos, o Sr. B passou a apresentar fantasias sexuais nas quais era um executivo que espancava, humilhava e abusava. Mais estudos centrados na psicopatologia das obsessões, dos impulsos e das compulsões nos parecem apropriados para que a relação entre estes fenômenos seja esclarecida.

Os casos 3 e 4 sugerem que pacientes com TOC e parafilias podem não lançar mão de comportamentos compulsivos na tentativa de anular ou de manter seus desejos, fantasias ou atos sexuais sob controle [os quais podem ser egossintônicos (caso 3)] ou mesmo sequer apresentar obsessões de conteúdo sexual dentre os seus sintomas obsessivo-compulsivos (casos 3 e 4). Os fatores que determinam o surgimento de compulsões "antifantasias" ou a transformação de obsessões sexuais egodistônicas em fantasias egossintônicas devem ser investigados em estudos futuros com amostras maiores.

Todos os pacientes descritos no presente relato apresentaram graves problemas quanto à adesão ao tratamento. As razões para este fenômeno são desconhecidas, mas podem incluir vergonha ou constrangimento diante do relato de seus sintomas sexuais aos profissionais responsáveis por seus cuidados clínicos. Estas vivências podem ser particularmente intensas em pacientes com TOC, sabidamente conscienciosos.

Fontenelle et al. (2005) sugeriram que pacientes com a comorbidade entre TOC e TCI requerem um maior número de ensaios terapêuticos com IRS até alcançar resposta terapêutica. Portanto, é possível que, mediante um incremento da adesão ao tratamento, alguns pacientes com TOC e parafilias respondam adequadamente à medicação instituída. De fato, embora alguns estudos sugiram que os IRS podem ser eficazes no manejo de pacientes com parafilias (Zohar et al., 1994; Abouesh e Clayton, 1999), tais medicações não demonstraram ser eficazes em outros pacientes, especialmente aqueles com sintomas parafílicos egossintônicos (Abdo et al., 2001).

De qualquer forma, a adesão ao tratamento se torna ainda mais importante diante das evidências de que algumas classes de drogas podem ser eficazes, tanto no TOC resistente ao tratamento quanto nas parafilias, incluindo o acetato de ciproterona (Casas et al., 1986; Feldman et al., 1988; Gijs e Gooren, 1996), os hormônios liberadores da gonadotrofina [por exemplo, triptorelina (Eriksson, 2000; Eriksson, 2007; Briken et al., 2003)] e alguns anticonvulsivantes [por exemplo, topiramato (Khazaal e Zullino, 2006; Marazziti e Dell'Osso, 2006; Hollander e Dell'Osso, 2006; Van Ameringen et al., 2006)].

\section{CONCLUSÃO}

Nosso estudo sugere que TOC e parafilias podem se associar em um mesmo paciente, observação esta inconsistente com a visão de que compulsividade e impulsividade devem ser consideradas pólos opostos do espectro compulsivoimpulsivo. Nosso estudo sugere que (1) pacientes com TOC e transtornos parafílicos tendem a desenvolver o TOC primeiro, (2) diante de desejos, fantasias ou atos sexuais parafílicos, pacientes com TOC podem lançar mão de comportamentos tipicamente compulsivos, (3) pacientes com TOC e obsessões sexuais egodistônicas podem desenvolver desejos, fantasias ou atos sexuais parafílicos, (4) em um mesmo paciente, TOC e parafilias podem apresentar cursos independentes e (5) pacientes com TOC e parafilias podem não apresentar obsessões sexuais. Pacientes com TOC e transtornos parafílicos apresentam pouca adesão ao tratamento por razões ainda desconhecidas. O sofrimento de pacientes com TOC e parafilias justifica a investigação continuada de tais condições no intuito de elucidar claramente os mecanismos que subjazem esta associação e de criar estratégias que aumentem a adesão ao tratamento. 


\section{REFERÊNCIAS}

Abdo CH, Hounie A, de Tubino Scanavino M, Miguel EC. OCD and transvestism: is there a relationship? Acta Psychiatr Scand, 103(6):471-3, 2001.

Abouesh A, Clayton A. Compulsive voyeurism and exhibitionism: a clinical response to paroxetine. Arch Sex Behav, 28(1):23-30, 1999 .

Briken P, Hill A, Berner W. Pharmacotherapy of paraphilias with long-acting agonists of luteinizing hormone-releasing hormone: a systematic review. J Clin Psychiatry, 64(8):890-7, 2003.

Carlsson ML. On the role of prefrontal cortex glutamate for the antithetical phenomenology of obsessive compulsive disorder and attention deficit hyperactivity disorder. Prog Neuropsychopharmacol Biol Psychiatry, 25(1):5-26, 2001.

Casas M, Alvarez E, Duro P, Garcia-Ribera C, Udina C, Velat A, et al. Antiandrogenic treatment of obsessive-compulsive neurosis. Acta Psychiatr Scand, 73(2):221-2, 1986.

Eriksson T. Antiandrogenic treatment for obsessive-compulsive disorder. Am J Psychiatry. 2000;157(3):483

Eriksson T. Anti-androgenic treatment of obsessive-compulsive disorder: an open-label clinical trial of the long-acting gonadotropin-releasing hormone analogue triptorelin. Int Clin Psychopharmacol, 22(1):57-61, 2007.

Feldman JD, Noshirvani H, Chu C. Improvement in female patients with severe obsessions and/or compulsions treated with cyproterone acetate. Acta Psychiatr Scand, 78(2):254, 1988.

Fontenelle LF, Mendlowicz MV, Versiani M. Impulse control disorders in patients with obsessivecompulsive disorder. Psychiatry Clin Neurosci, 59(1):30-7, 2005

Fontenelle LF, Mendlowicz MV, Marques C, Versiani M. Early- and late-onset obsessive-compulsive disorder in adult patients: an exploratory clinical and therapeutic study. J Psychiatr Res. 2003;37(2):127-33.

Giij L, Gooren L. Hormonal and psychopharmacological interventions in the treatment of paraphilias: an update. J Sex Res, 33:273-290, 1996.
Grant JE. Clinical characteristics and psychiatric comorbidity in males with exhibitionism. J Clin Psychiatry, 66(11):1367-71, 2005.

Grant JE, Mancebo MC, Pinto A, Eisen JL, Rasmussen SA. Impulse control disorders in adults with obsessive compulsive disorder. J Psychiatr Res, 40(6):494-501, 2006.

Hollander E, Dell'Osso B. Topiramate plus paroxetine in treatment-resistant obsessive-compulsive disorder. Int Clin Psychopharmacol, 21(3):189-91, 2006.

Hollander E, Wong CM. Obsessive-compulsive spectrum disorders. J Clin Psychiatry, 56(4 Suppl):3-6, 1995

Khazaal Y, Zullino DF. Topiramate in the treatment of compulsive sexual behavior: case report. BMC Psychiatry, 23;6:22, 2006.

Marazziti D, Dell'Osso B. Topiramate plus citalopram in the treatment of compulsive-impulsive sexual behaviors. Clin Pract Epidemol Ment Health, 22;2:9, 2006.

Matsunaga H, Kiriike N, Matsui T, Oya K, Okino K, Stein DJ. Impulsive disorders in Japanese adult patients with obsessive-compulsive disorder. Compr Psychiatry, 46(1):43-9, 2005.

Rosario-Campos MC, Leckman JF, Mercadante MT, Shavitt RG, Prado HS, Sada P, Zamignani D, Miguel EC. Adults with early-onset obsessive-compulsive disorder. Am J Psychiatry. 2001;158(11):1899-903

Stein DJ, Hollander E, Simeon D, Cohen L. Impulsivity scores in patients with obsessive-compulsive disorder. J Nerv Ment Dis, 182(4):240-1, 1994.

Stewart SE, Jenike MA, Keuthen NJ. Severe obsessive-compulsive disorder with and without comorbid hair pulling: comparisons and clinical implications. J Clin Psychiatry, 66(7):864-9, 2005.

Van Ameringen M, Mancini C, Patterson B, Bennett M. Topiramate augmentation in treatmentresistant obsessive-compulsive disorder: a retrospective, open-label case series. Depress Anxiety, 23(1):1-5, 2006

Zohar J, Kaplan Z, Benjamin J. Compulsive exhibitionism successfully treated with fluvoxamine: a controlled case study. J Clin Psychiatry, 55(3): 86-8, 1994. 